\title{
Trustworthy Device Pairing for Opportunistic Device-to-Device Communications in Mobile Crowdsourcing Systems
}

\author{
Cong Zhao \\ Xi'an Jiaotong University \\ Fengrui Shi \\ Xi'an Jiaotong University \\ zhaocong@stu.xjtu.edu.cn arthur.shifengrui@gmail.com xjhznick@gmail.com \\ Xinyu Yang \\ X'an Jiaotong University \\ yxyphd@mail.xjtu.edu.cn \\ Julie McCann \\ Imperial College London \\ j.mccann@imperial.ac.uk
}

\begin{abstract}
Mobile Crowdsourcing System is an emerging service paradigm base on numerous personal smart devices, where the Deviceto-Device communication among opportunistically encountered participating devices is an indispensable part of task allocation, file transmission and data collaboration. Considering that participating devices are privately held and opportunistically encountered, we design the Trustworthy Device Pairing (TDP) scheme that realizes user-transparent sharing secret key negotiation and reliable peer device determination for trustworthy spontaneous D2D transactions. TDP is demonstrated to be effective based on our proof-of-concept implementation, and a further evaluation on efficiency will be conducted.
\end{abstract}

\section{INTRODUCTION}

Mobile devices with enriched sensing capabilities stimulate the proliferation of mobile crowdsourcing systems (MCSs), an ubiquitous, heterogenous and smart service paradigm that aims to provide data collecting, processing and computing services, by exploiting the capacities of ubiquitous personal mobile devices in a collaborative way [1].

The emerging Device-to-Device (D2D) communication techniques [2] such as WiFi Direct, Bluetooth Smart, and LTE direct have a great potential to provide cost-effective solutions to collaborative interactions among mobile devices for mobile crowdsourcing [3,4]. However, since participating devices are private and temporarily hired, there is no prior trust relationship between devices opportunistically encountered. The initial trust establishment, or Device Pairing, cannot rely on physical interactions between unfamiliar device holders. Furthermore, considering the high device density, there is no reference to determinate a proper peer device among multiple available candidates.

Permission to make digital or hard copies of all or part of this work for personal or classroom use is granted without fee provided that copies are not made or distributed for profit or commercial advantage and that copies bear this notice and the full citation on the first page. Copyrights for components of this work owned by others than ACM must be honored. Abstracting with credit is permitted. To copy otherwise, or republish, to post on servers or to redistribute to lists, requires prior specific permission and/or a fee. Request permissions from Permissions@ acm.org.

S3'15, September 11, 2015, Paris, France.

Copyright 2015 ACM. ISBN 978-1-4503-3701-4/15/09 ...\$15.00.

DOI: http://dx.doi.org/10.1145/2801694.2801705.
In this paper, we propose a mechanism, TDP, for opportunistically encountered devices in MCSs to autonomously establish a secure D2D connection transparently to device holders. Moreover, trustworthy peer device is determined based on the device trust value reflecting the reliability on fulfilling different types of D2D transactions. Specifically:

- TDP realizes user-transparent device paring that is not supported by off-the-shelf protocols $[5,6]$ and rapid device paring that is faster than state-of-art non-interactive device pairing schemes [7-9], and device trustworthy is novelly adopted for the determination of the most reliable D2D peer device.

- TDP guarantees a comparable security intensity as offthe-shelf device pairing protocols $[5,6]$ while taking account of traffic oriented attacks, like the Trust Forging Attack and the Collusive Attack manipulating D2D traffic, that are exclusive for D2D communications in MCSs.

\section{SYSTEM ARCHITECTURE}

An MCS, see Fig.1(a), is generally composed of a Backend Server (BS) and numerous participating devices $(A, B, \ldots, N)$. Specifically, BS formulates service campaigns, publishes crowdsourcing applications, outsources computing and sensing tasks, manages feedback and maintains device profiles. On the other hand, any personal smart device that has installed the crowdsourcing application and registered at BS becomes a potential contributor to crowdsourcing tasks. Devices communicate with BS through wireless access of the Internet, like WiFi or cellular access points, meanwhile they communicate with each other in a D2D manner through outband wireless channels, like Bluetooth or WiFi Direct connections.

Based on the crowdsourcing application installed on participating devices, BS outsources crowdcomputing tasks intending to offload computing or processing intensities and crowdsensing tasks intending to spontaneously collect environmental or social status data. Accepting allocated tasks, participating devices need to establish D2D connections with opportunistically encountered peers to recruit collaborators, exchange intermediate processing results, or forward sensing data packets. 


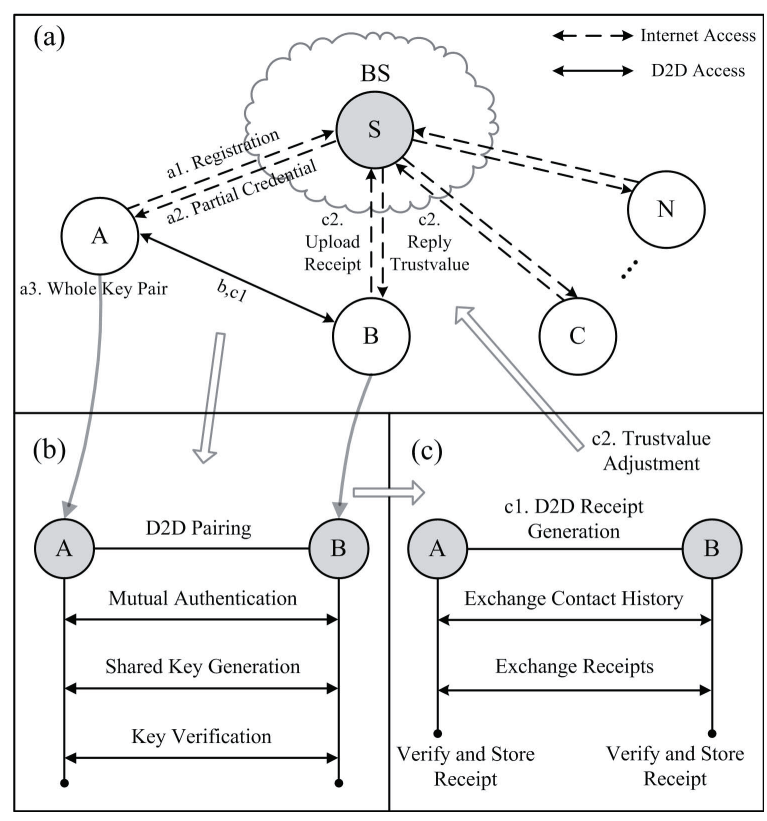

Figure 1: Architecture of TDP

\section{TRUSTWORTHY DEVICE PAIRING}

Considering issues discussed in Section 1, we design the Trustworthy Device Pairing (TDP) scheme to realize opportunistic D2D communications and the D2D trust management in MCSs. Specifically, it has three major components: Device Registration, Device Pairing, and Device Trustvalue Management, whose general procedure is illustrated in Fig.1

\subsection{Device Registration}

In our design, device pairing credentials are generated and managed within a Certificateless Public Key Cryptography (CL-PKC) framework [10], where an authenticated key pair composed of a private key and a public key $(s k, p k)$ is utilized for device authentication and sharing secret negotiation. Under CL-PKC settings, the Computational DiffieHellman Problem on the Elliptic Curves [11] is treated as an intractable mathematical problem.

For the system initialization, BS setups security parameters according to system scale, like expected user amount, server capacities, and types of provided services. An elliptic curve $\mathbf{E}$ and the master key pair $\left(x, P_{p u b}\right)$ of $\mathrm{BS}$ is generated, where $\mathbf{E}$ and $P_{p u b}$ are predeployed into our crowdsourcing application.

Any personal smart device, says A, that has installed the application becomes a potential crowdsourcing task contributor. To get its D2D credential, A needs to register at BS. The secret and public key pair of $\mathrm{A}\left(s k_{a}, p k_{a}\right)$ is cooperatively generated by $\mathrm{A}$ and $\mathrm{BS}$, where parts of $s k_{a}$ and $p k_{a}$ are locally generated by $\mathrm{A}$ and the rest of them is generated by BS based on the received public key part of A using the master secret key $x$. Such a cooperative means guarantees that BS is not able to decrypt D2D traffic between any arbitrary pair of encountered devices since it does not have any entire key pair, while, generated using the master secret key $x$, any valid key pair itself can be treated as an authenticated certificate for mutual authentication between opportunistically encountered devices.

\subsection{Device Pairing}

The determination of a proper peer when there are multiple options will be explicitly discussed in the next section. Without loss of generality, when determines to conduct a $\mathrm{D} 2 \mathrm{D}$ transaction with registered device $\mathrm{B}$, registered device A will initiate the Device Pairing process in Fig.1(b).

With the public key of peer device in the probe packet, $\mathrm{A}$ and $\mathrm{B}$ will respectively generate a partial sharing secret $V_{a(b)}$ based on their own private key $s k_{a(b)}$, the other's public key $p k_{b(a)}$ and the main public key $P_{p u b}$. Since the partial sharing secret based on one's public key can only be verified using corresponding private key, and valid public keys of registered devices can be authenticated using the main public key, A and B are able to restore the partial sharing secret of their peer device $V_{b(a)}^{\prime}$.

The final sharing secret $S_{a b}=V_{a} \oplus V_{b}^{\prime}=V_{a}^{\prime} \oplus V_{b}$ can be authenticated using general Challenge-Response schemes. If $S_{a b}$ is successfully established, then we say A and B are mutually authenticated, and $S_{a b}$ is treated as the session key of the current D2D transaction.

\subsection{Device Trustvalue Management}

In our design, we use behaviors in previous D2D transactions to evaluate the reliability of device fulfilling future transactions, which is regarded as the criterion of the peer device determination when there are multiple D2D options.

We define such a reliability as Device Trustvalue $\boldsymbol{t}\left(I D_{a}, i\right)$, which is an $n$-dimension vector with the value of each dimension representing the reliability of device A fulfilling corresponding type of D2D transaction defined by BS after its $i$ 'th transaction. $\boldsymbol{t}\left(I D_{a}, i\right)$ can be calculated as

$$
\boldsymbol{t}\left(I D_{a}, i\right)=\frac{2}{\pi} \arctan \left(\tan \left(\frac{\pi}{2} t\left(I D_{a}, i-1\right)\right)+\Delta \boldsymbol{t}\right)
$$

where $\Delta \boldsymbol{t}$ is defined as

$$
\Delta \boldsymbol{t}=S * C r e * \boldsymbol{t} \boldsymbol{v},
$$

where $S$ is the feedback A received in its $i$ th D2D transaction, $\boldsymbol{t} \boldsymbol{v}$ is an $n$-dimension vector that represent the weight of such a transaction, and Cre is A's confidence level on $S$, which is defined as

$$
\text { Cre }=\beta \text { Div }+(1-\beta) \text { Sim, }
$$

where Div denotes the relative diversity of devices that A and its peer have paired with before, Sim denotes the similarity of feedback that respectively from A and its peer to devices that both of them have paired with before. Both of them can be retrieved from device pairing histories that record previous feedback to those paired devices. $\beta \in(0,1)$ is the weight adjustment coefficient. The meaning of Div and Sim is to prevent potential adversaries from forging their own trustvalues or collusively enhancing each other's trustvalue within a group to manipulate D2D traffic.

With such a definition of device trustvalue, according to Fig.1(c), a participating device, says A, will cooperatively generate a $\mathrm{D} 2 \mathrm{D}$ transaction receipt $R e$ with its peer device, says B, to record feedback, credibility and transaction vector for a trustvalue adjustment at BS. Such a receipt is, and only is, verifiable to $\mathrm{A}, \mathrm{B}$ and $\mathrm{BS}$ using their private keys, while the obfuscated feedback is verifiable to the receiver without revealing its actual value.

For participating devices, the trustvalue adjustment is delay-tolerant, which is only performed when there is an 


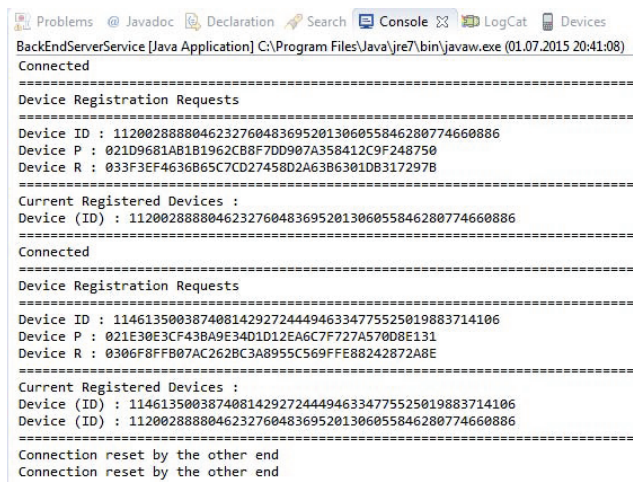

Figure 2: Implementation of BS

economical access to the Internet. BS is responsible for the verification of D2D receipts and the adjustment of trustvalues, after which it will generate a corresponding digital signature of the adjusted trustvalue for its future D2D paring authentication.

\section{IMPLEMENTATION}

We implement a Proof-of-Concept version of device registration and pairing functionalities on a prototype consisting of a BS based on Window7 workstation and two Android smart devices (Google Nexus7 with Android L and Samsung Galaxy Note3 with Andorid 4.3 Jelly Bean) with Bluetooth V4.0 modules, where our implementation is running as a security service upon a rapid insecure Bluetooth connection [12].

With cryptography primitives introduced by [13] and ECC parameters adopted from secp160r1 [14], device registration and pairing functionalities are realized, as shown in Fig.2 and 3. D2D credentials $s k=(x, d), p k=(P, R)$ are cooperatively generated by $\mathrm{BS}$ and the participating device. After the registration, device is able to start the crowdsourcing service that allows user-transparent pairing with other registered device encountered, and the D2D session key $S K$ will be negotiated and authenticated based on D2D credentials.

\section{CONCLUSION AND FUTURE WORK}

In this paper, we presented TDP focusing on trustworthy device pairing for opportunistic D2D communications in MCSs. We realized a user-transparent device pairing scheme to establish sharing keys securing D2D transactions between encountered devices, then proposed a device trust management mechanism to evaluate the reliability of encountered devices fulfilling pending D2D transactions. Based on our implementation, TDP is demonstrated to be effective for MCSs.

In future, we will extend our paring scheme to WiFi Direct communications for a higher bandwidth, and further evaluate the performance of our trust management mechanism with both $\mathrm{OMNeT}++$ simulations and voluntary participating experiments in real campus environments. Additional factors we emphasize during the evaluation include participator mobility modeling and crowdsourcing task/D2D transaction modeling.

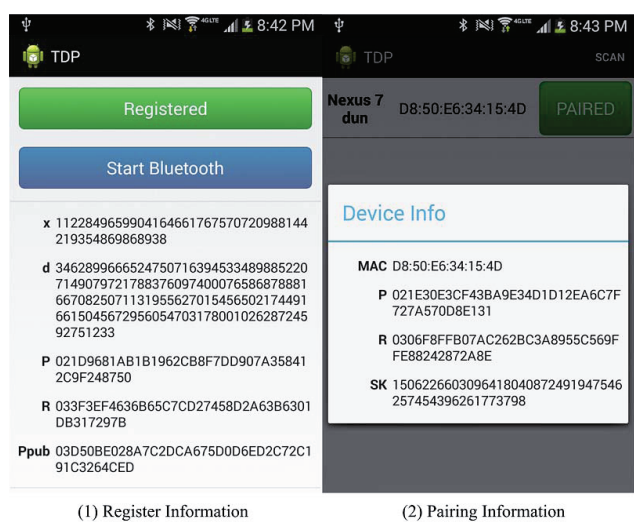

Figure 3: Device Registration and Pairing

\section{REFERENCES}

[1] Ju Ren, Yaoxue Zhang, Kuan Zhang, and Xuemin Shen. Exploiting mobile crowdsourcing for pervasive cloud services: challenges and solutions. IEEE Commun. Mag., 53(3):98-105, 2015.

[2] A. Asadi, Qing Wang, and V. Mancuso. A survey on device-to-device communication in cellular networks. IEEE COMMUN SURV TUT, 16(4):1801-1819, 2014.

[3] Shusen Yang, Usman Adeel, and Julie McCann. Selfish mules: Social profit maximization in sparse sensornets using rationally-selfish human relays. IEEE J. Sel. Areas Commun., 31(6):1124-1134, 2013.

[4] Shusen Yang, Usman Adeel, and Julie McCann. Backpressure meets taxes: Faithful data collection in stochastic mobile phone sensing systems. In Proc. IEEE INFOCOM, 2015.

[5] Wi-fi peer-to-peer (p2p) technical specification v1.5. Wi-Fi Alliance, 2014

[6] Bluetooth core specification 4.2. Bluetooth SIG, 2014.

[7] Markus Miettinen, N. Asokan, Thien Duc Nguyen, Ahmad-Reza Sadeghi, and Majid Sobhani. Context-based zero-interaction pairing and key evolution for advanced personal devices. In Proc. ACM CCS, pages 880-891. ACM, 2014.

[8] Hien Thi Thu Truong, Xiang Gao, Babins Shrestha, Nitesh Saxena, N Asokan, and Petteri Nurmi. Comparing and fusing different sensor modalities for relay attack resistance in zero-interaction authentication. In Proc. IEEE PerCom, pages 163-171. IEEE, 2014.

[9] Jingchao Sun, Xu Chen, Jinxue Zhang, Yanchao Zhang, and Junshan Zhang. Synergy: A game-theoretical approach for cooperative key generation in wireless networks. In Proc. IEEE INFOCOM, pages 997-1005. IEEE, 2014.

[10] Sattam S Al-Riyami and Kenneth G Paterson. Certificateless public key cryptography. In Advances in Cryptology-ASIACRYPT 2003, pages 452-473. Springer, 2003.

[11] Antoine Joux and Kim Nguyen. Separating decision diffie-hellman from computational diffie-hellman in cryptographic groups. Springer J CRYPTOL, 16(4):239-247, 2003.

[12] Android. Bluetoothadapter. http://developer.android.com/reference/android/ bluetooth/BluetoothAdapter.html.

[13] Bouncy Castle. The legion of the bouncy castle java cryptography apis. http://www . bouncycastle.org/java.html.

[14] Certicom Research. SEC 2: Recommended Elliptic Curve Domain Parameters. Standards for Efficient Cryptography Version 1.0, 2000. 